\title{
Caracterización Psicosocial y Salud Mental en Familias de Escolares Chilenos durante el Aislamiento Físico por la Covid-19
}

\section{Psychosocial Characterization and Mental Health in Families of Chilean Students during Physical Isolation by Covid-19}

\author{
Fabiola Sáez-Delgado ${ }^{1}$ * \\ Constanza Olea-González ${ }^{1}$ \\ Javier Mella-Norambuena ${ }^{2}$ \\ Yaranay López-Angulo ${ }^{3}$ \\ Héctor García-Vásquez ${ }^{1}$ \\ Rubia Cobo-Rendón ${ }^{4}$ \\ Felipe Sepúlveda López ${ }^{1}$ \\ ${ }^{3}$ Universidad Santo Tomás, Chile \\ ${ }^{4}$ Universidad San Sebastián, Chile
}

${ }^{1}$ Universidad Católica de la Santísima Concepción, Chile

${ }^{2}$ Universidad Técnica Federico Santa María, Chile

\begin{abstract}
Los objetivos del estudio fueron caracterizar variables psicosociales y académicas en familias de escolares de primaria y secundaria del sur de Chile en aislamiento físico y analizar la salud mental de padres y madres. Se utilizó un diseño descriptivo transversal. Se consideraron dos etapas. En la primera se aplicó una encuesta ad-hoc a 8.102 familias. En la segunda se aplicó el instrumento DASS-2 1 a una muestra de 59. Los resultados de la primera etapa evidenciaron en las familias que el $40 \%$ tenía un integrante sin trabajo remunerado, un ingreso menor a $\$ 440$, y la gran mayoría no disponía de computador. Los escolares han sentido ansiedad, miedos nocturnos, mal humor o agresividad, varios no han realizado tareas escolares y reportaron dificultades motivacionales, la mayoría no ha dedicado suficiente tiempo al estudio y se han dedicado a ver series. En la segunda etapa, el $57,6 \%$ de los padres y madres presentó ansiedad severa, el 50,8\% estrés y un $83 \%$ depresión. Se concluye que las familias carecen de recursos para el desarrollo efectivo de la educación en línea. Se requieren acciones especializadas para "mitigar" el impacto del aislamiento físico por Covid-19 en variables socio-académicas y de salud mental en escolares y sus familias.
\end{abstract}

Descriptores: Escuela; Educación en línea; Educación primaria; Familia; Justicia social.

The research objectives were to examine psychosocial and academic variables in families of primary and secondary schoolchildren in southern Chile in physical isolation and to analyze the mental health of parents. A descriptive cross-sectional design was used. Two stages were considered. In the first stage, an ad-hoc survey was applied to 8,102 families. In the second stage, the DASS-2 1 instrument was applied to a sample of 59. The results of the first stage showed that $40 \%$ of the participant families had a member without paid work, an income of less than $\$ 440$, and the majority did not have computer at home. The schoolchildren have felt anxiety, night fears, bad mood or aggressiveness, several of them have not completed schoolwork and reported motivational difficulties. Most of surveyed students have not dedicated enough time to schoolwork and their main activity has been to watch TV series. The second stage showed that the $57.6 \%$ of parents presented severe anxiety, $50.8 \%$ stress and $83 \%$ depression. It is concluded that families lack the resources for the effective development of online education. Specialized actions are required to "mitigate" the impact of physical isolation by Covid-19 on socio-academic and mental health variables in schoolchildren and their families.

Keywords: School; Online education; Primary education; Families; Social justice.

*Contacto: fsaez@ucsc.cl

ISSN: 2254-3139

www.rinace.net/riejs/

revistas.uam.es/riejs
Recibido: $\quad 15$ de julio 2020

$1^{\text {a }}$ Evaluación: 13 de agosto 2020

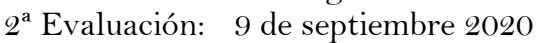

Aceptado: $\quad 7$ de noviembre 2020 


\section{Introducción}

La Covid-19 originada en diciembre de 2019, ha tenido consecuencias negativas para la sociedad y la amenaza sigue latente (Yi et al., 2020). El primer caso "latinoamericano" de coronavirus fue informado en Brasil el 26 de febrero de 2020 y la primera muerte causada por la infección en la región se avisó en Argentina el 7 de marzo (Pierre y Harris, 2020). En Chile, se informó el primer caso confirmado de coronavirus el 3 de marzo de 2020 (Minsal, 2020). La Organización Mundial de la Salud (OMS) declaró la aparición de la enfermedad como pandemia, causado por el síndrome respiratorio agudo severo coronavirus (SARS-CoV-2) con fecha 12 de marzo de 2020 (Viner et al., 2020).

La acelerada propagación de la Covid-19 en América, se observa en la proliferación de contagios diarios en cada país y por la velocidad de expansión; esto ha hecho que cada sector de la vida humana sienta su impacto (Sintema, 2020). Como consecuencia, se ha instalado en las personas una profunda incertidumbre sustentada en la falta de claridad y desconocimiento del tiempo que se extenderá la pandemia; aún se desconoce cuántas víctimas alcanzará y las complicaciones que conllevará, considerando la ausencia de una vacuna o bien un tratamiento específico (Pierre y Harris, 2020). Una de las medidas implementadas como respuesta inmediata para frenar el contagio, ha sido velar por el distanciamiento físico; implicando para cada persona romper con las actividades habituales de la vida cotidiana.

El presente estudio emerge a partir de la falta de evidencia sobre las características psicosociales de escolares y el estado de salud mental de padres y madres en tiempos de aislamiento físico por la pandemia Covid-19. Disponer de resultados específicos aportará a mejorar la educación en medio de esta circunstancia y postcovid-19 dado que, contar con evidencia empírica orientará la toma de decisiones (Murillo, 2016). Es oportuno este estudio, en tanto, investigaciones en Latinoamérica respecto de las características de integrantes en las comunidades educativas durante la pandemia Covid-19 son escasas.

\section{Revisión de la literatura}

La alteración de la cotidianidad ha creado una situación insólita para las organizaciones y los establecimientos educativos; se han visto obligados a la suspensión de actividades académicas presenciales (Sánchez et al., 2020; Viner et al., 2020). El cierre de instituciones educativas está afectando la educación del $80 \%$ de escolares en todo el mundo (Van Lancker y Parolin, 2020). La obligatoria suspensión de las clases en todos los niveles educativos, ha implicado una red de efectos en múltiples características de docentes, familias y escolares (Sánchez et al., 2020).

A nivel mundial los países han hecho giros estratégicos según sus condiciones técnicas y políticas para ejecutar las clases no presenciales en una transición rápida y obligada a la educación a distancia, mediada por tecnologías (Villafuerte et al., 2020). El uso de las Tecnologías de la Información y Comunicación (TIC) ha sido la alternativa para minimizar los riesgos de contagio sin detener el avance de los estudios (Villafuerte et al., 2020), lo cual podría resaltar una diferenciación social respecto del acceso a la educación en función de los recursos de cada familia. Sin embargo, un resultado en España mostró que, aunque la mayor parte de estudiantes contaba con recursos tecnológicos en sus hogares durante la pandemia Covid-19, confirma que no se trata de que las familias dispongan de diferentes tecnologías, sino más bien, que los recursos tecnológicos 
posibiliten continuar con los estudios mientras no se pueda acudir a los centros educativos (Rodicio-García et al., 2020).

En Latinoamérica, la conectividad en el nivel educativo primario y secundario es precaria y desigual desde antes de la pandemia. La implementación de nuevas reformas educativas ha enfrentado dificultades significativas caracterizadas por la existencia de infraestructura deficiente, donde muchos países han tenido que optar entre dedicar los escasos recursos disponibles a satisfacer las necesidades básicas de equipamiento de las escuelas, nutrición y salarios docentes o invertir en los requerimientos propios del proceso de desarrollo tecnológico (Tedesco, 2014). De hecho, el estudio de las tecnologías en el campo de la educación se ha centrado a menudo en temas relacionados con los beneficios que estas tienen en los procesos de aprendizaje, pero no consideran que los sistemas escolares están marcados por la desigualdad en el acceso a oportunidades de aprendizaje en línea, inclusive las intervenciones con tecnología pueden también tener efectos diferenciales que favorecen a los grupos acomodados y podría ampliar la desigualdad social (Tawfik, Reeves y Stich, 2016).

La actual situación de pandemia está dirigiendo a las instituciones educativas a que algunas de las soluciones propuestas potencialmente puedan profundizar las desigualdades e injusticias sociales, pero que parecen verse soterradas en el contexto de crisis sanitaria por la Covid-19 y la necesidad de dar una solución inmediata, en lugar de una solución profunda y efectiva para todas y todos. La capacidad de respuesta a la pandemia ha dependido de elementos psicosociales que determina los recursos concretos de las y los integrantes de una comunidad educativa; el acceso a las TIC y la cotidianidad de su uso revela un estatus de acceso a la educación, derecho básico de los seres humanos, donde su distribución diferenciada da cuenta de una brecha de desigualdad crítica desestimada hasta el momento (Lusquiños, 2020).

La enseñanza mediada por las TIC mostró con fuerza las desigualdades en el acceso a la educación y particularmente en el acceso a la educación de calidad; la Covid-19 ha significado una nueva desventaja para la educación de las y los estudiantes de sectores socioeconómicos bajos, medios-bajos y medios, profundizando la desigualdad en educación al acceder con mayores limitaciones a la educación virtual (Quiroz, 2020). El cambio en la interacción entre estudiantes y profesores ha puesto de manifiesto necesidades previas y contingentes de los establecimientos, en cuanto a infraestructura y preparación del capital humano. La enseñanza en línea visibiliza desigualdades sociales sobre todo vinculadas a las posibilidades de recursos para la conectividad.

En Chile, a nivel escolar primario y secundario, se siguen indicaciones gubernamentales por la consecuente activación de una serie de protocolos, que ha implicado transitar de la educación presencial a modalidades no presenciales mediadas por las tecnologías; siendo difícil la adaptación a la virtualidad. El Ministerio de Educación chileno ha promovido diferentes herramientas que facilitan dar continuidad con el proceso educativo tales como: la utilización de programas de televisión abierta con enfoque educativo, envío de guías virtuales, materiales de estudios en formato impreso y plataformas en línea para la continuación de los aprendizajes. Una de las dificultades que se han visualizado ha sido la disposición y preparación de las familias para acompañar a las y los escolares en la teleducación en confinamiento, lo cual abre brechas entre los grupos sociales (Quiroz, 2020). En tal sentido, para alcanzar los objetivos de aprendizaje, la alianza entre familias, docentes y las y los escolares es la clave (Cáceres, 2020). 
Ya se advierte sobre la probabilidad de una caída en el porcentaje de aprobación de estudiantes de secundaria en los exámenes nacionales de este año por las modificaciones al calendario académico por la Covid-19 (Sintema, 2020). Este resultado invita a reflexionar sobre el tiempo de trabajo pedagógico por parte de los profesores, y la necesidad de que las familias hagan un trabajo de mediación efectivo en el proceso de enseñanza-aprendizaje entre estudiantes y profesores.

La educación en línea en estas circunstancias plantea el desafío de generar vínculos más estrechos entre los integrantes de la comunidad educativa, generando un buen ambiente para que los estudiantes aprendan de manera eficiente (Zhou et al., 2020). La participación de las familias es clave para llevar a buen término los objetivos educativos que se propongan, para esto se requiere voluntad y comunicación entre los involucrados, con el fin de evitar tensiones entre integrantes de la comunidad educativa sobre todo entre expectativas de padres y docentes; además, es necesario conocer cómo se desarrolla la convivencia en las familias, qué desafíos presentan en torno a la conectividad con profesores y en el desarrollo de las tareas escolares para proseguir con la enseñanza desde la casa (Cervantes y Gutiérrez, 2020).

Sin embargo, se han identificado tensiones entre las expectativas de las familias y escolares; así, docentes se enfrentan a una participación limitada de las familias, quienes además la perciben como impuesta (Holguín y Sandoval, 2020); dichas tensiones asociadas a la educación, conjuntamente con la incertidumbre circunstancial, el confinamiento y las diferentes exigencias que enfrenta cada ser humano; han traído consecuencias para la salud mental de estudiantes, familias y profesores. La evidencia empírica muestra que las medidas de respuesta común a la pandemia Covid-19 adoptadas por los diferentes países, como la distancia social, el encierro y el quedarse en casa, tienen un impacto significativo y negativo en el bienestar de las personas (Zolotov et al., 2020).

Las dificultades en la salud mental y el bienestar pueden ser graves en la mayoría de escolares; la migración forzada a la enseñanza en línea les ha generado ansiedad, lo cual debe aliviarse para garantizar que puedan participar de manera activa y efectiva en el aprendizaje en esta modalidad (Bao, 2020).

Estudios previos al desenlace de la pandemia por Covid-19 han evidenciado que la salud mental de padres y madres es afectada por diversos factores, por ejemplo, que sus hijos o hijas necesiten apoyos escolares adicionales (Ang y Loh, 2019). Así también, específicamente en latinos, se ha demostrado que padres y madres experimentan mayor estrés por factores como la aculturación (Lorenzo-Blanco et al., 2016) o por problemas financieros (Gilbert, Spears y Mistry, 2017). Esto a su vez, tiene un impacto sobre la salud mental, el abuso en el consumo de sustancias y el adecuado funcionamiento familiar, además de disminuir la participación en el monitoreo del desempeño académico de sus hijos e hijas (Gilbert, Spears y Mistry, 2017; Lorenzo-Blanco et al., 2016), influyendo en el rendimiento académico de los estudiantes (Lui et al., 2019).

Durante la pandemia de Covid-19, un estudio en padres y madres en China mostró que los factores de protección a la salud mental eran las buenas relaciones maritales, el apoyo social, familias armoniosas y sin antecedentes de enfermedad mental; mientras que el estrés percibido y tener hijos o hijas en nivel escolar fueron percibidos como factores de riesgos (Wu et al., 2020). Además, una revisión de la literatura existente sobre estudios de salud mental durante la Covid-19 sugiere que los síntomas de ansiedad y depresión (16$28 \%$ ) y el estrés autoinformado (8\%) han sido reacciones psicológicas comunes ante la 
pandemia (Rajkumar, 2020), consecuencias que deben ser exploradas en Latinoamérica, donde aún no se cuenta con suficiente evidencia empírica al respecto.

Por los antecedentes mostrados, se sugiere la realización de nuevas investigaciones que profundicen en aspectos psicosociales y de salud mental durante el aislamiento físico para aportar al ajuste de medidas socioeducativas hacia un adecuado desarrollo humano y promoción de aprendizajes. Debido a la situación actual se hace necesario conocer cómo se desarrolla la convivencia de las familias, qué desafíos presentan en torno a la conectividad con las y los docentes/escuela y en el desarrollo de las tareas escolares. Por tal motivo, esta investigación se propuso dos objetivos: (1) caracterizar variables psicosociales y académicas en familias de escolares de nivel primario y secundario del sur de Chile y (2) analizar variables asociadas a la salud mental de padres y madres.

\section{Método}

Esta investigación se realizó desde un enfoque cuantitativo. Se consideraron dos etapas consecutivas para responder a los objetos específicos uno y dos respectivamente. El alcance del estudio fue exploratorio-descriptivo donde se describen las variables de interés tal y como se dan en su medio natural, sin manipular. Se realizó la identificación de las características de acceso, educativas y de salud mental de las y los estudiantes y sus padres y madres (Ato, López y Benavente, 2013).

La muestra fue por conveniencia y accesibilidad. La primera etapa estuvo constituida por una muestra de 8.102 familias, de los cuales 6.077 escolares se encontraban en la etapa de primaria y 2.025 restantes, se encontraban cursando el nivel secundario. Del total de la muestra encuestada 6.129 eran mujeres (76\%) y 1.973 eran hombres (24\%). La muestra es representativa de la población $(79,61 \%)$, dado que la comunidad escolar matriculada en establecimientos municipales de la comuna de la zona centro sur de Chile descrita, es de un total de 10.177 estudiantes; representado en 33 establecimientos (26 de primaria y 7 de secundaria), de todos los establecimientos hubo participación. El índice de vulnerabilidad escolar (IVE) para el total de establecimientos reportado por la Junta Nacional de Auxilio Escolar y Becas del Estado de Chile es de 91,7\% (JUNAEB, 2020).

De la muestra anterior, participaron en una segunda etapa de medición 59 padres y madres, de estos, 4 eran hombres $(6,8 \%)$ y 55 eran mujeres $(93,2 \%)$ con una edad promedio de 45 años $(\mathrm{DE}=7,13)$, el nivel educativo en su mayoría era de secundaria $(91,1 \%)$, y de éstos, el $61 \%$ se encontraba en situación de desempleo.

En la primera etapa de este estudio, se utilizó una encuesta ad-hoc, la cual fue aplicada para levantar información sobre aspectos psicosociales y académicos en el periodo inicial de aislamiento físico por la Covid-19. La encuesta está compuesta por cuatro categorías principales durante el aislamiento físico por Covid-19 correspondientes a los siguientes aspectos: (1) sociofamiliares, con 7 preguntas; (2) conectividad, con 3 preguntas; (3) salud mental, con 3 preguntas; y (4) actividades escolares, con 4 preguntas. Las variables referidas a elementos económicos fueron transformadas desde pesos chilenos a dólares americanos y representan niveles de ingreso mensual.

En la segunda etapa de este estudio, se utilizó el DASS-2 1 para la medición de los niveles de salud mental. El DASS-2 1 tiene 21 ítems con el objetivo de evaluar tres dimensiones: depresión, ansiedad y estrés ( 7 ítems cada escala) y ha sido validado en población chilena (Antúnez y Vinet, 2012). La dimensión de estrés tiene un alfa de $\alpha=0,83$, un ejemplo de 
ítems es "Reaccioné exageradamente en ciertas situaciones". La dimensión de depresión tiene un alfa de $\alpha=0,85$, un ejemplo de ítems es "Sentí que la vida no tenía ningún sentido" y la dimensión de ansiedad tiene un alfa de $\alpha=0,73$, un ejemplo de ítems es "Se me hizo difícil respirar". Es un instrumento de autoinforme que tiene cuatro opciones de respuesta en formato tipo Likert ( 0 = "No describe nada de lo que me ha pasado" hasta $3=$ "Sí, esto me ha pasado mucho, o casi siempre"). Para responder las escalas, los padres y madres leían la consigna "indique en qué medida la frase describe lo que le pasó o sintió desde el inicio del confinamiento físico por la Pandemia Covid-19". El DASS-2 1 es considerado un instrumento con sólida consistencia interna y provee una adecuada distinción entre ansiedad y depresión, en comparación con otras medidas existentes (Antúnez y Vinet, 2012).

Para la identificación de medidas de salud mental se realiza la suma de los ítems correspondientes a cada dimensión, Depresión (ítems: 3, 5, 10, 13, 16, 17 y 21), Ansiedad (ítems: 2, 4, 7, 9, 15, 19 y 20) y Estrés (ítems: 1, 6, 8, 11, 12, 14 y 18). Posteriormente se identifican las puntuaciones con los siguientes puntos de corte según cada dimensión, Depresión (5-6 leve; 7-10 moderada; 11-13 severa; 14 o más, extremadamente severa). Ansiedad (4 leve; 5-7 moderada; 8-9 severa; 10 o más, extremadamente severa); Estrés (89 leve; 10-12 moderado; 13-16 severo; 17 o más, extremadamente severo) (Orellana y Orellana, 2020).

\section{Procedimiento para la recogida y análisis de la información}

Se solicitó autorización a responsables de la Dirección de Administración de Educación Municipal, de establecimientos educativos y a familias encuestadas, quienes dieron su consentimiento para ser parte del estudio. La aplicación del instrumento correspondiente a la etapa 1 (encuesta) se realizó a través de diversas plataformas digitales, llamadas telefónicas, WhatsApp, correo electrónico, Facebook institucional y otros. Este fue aplicado por los profesores jefes de los diferentes cursos desde el 23 de marzo al 10 de abril del año 2020 a 33 establecimientos educacionales. Los instrumentos de la etapa 2 fueron aplicados presencialmente por psicólogos. Se les instruyó siguiendo los protocolos de seguridad sanitaria establecidos por autoridades y por el Prevencionista de Riesgos de la Dirección de Administración de Educación Municipal. Se les entregó todo el equipo de prevención exigido. La encuesta fue realizada respetando el distanciamiento físico y no ingresando a los domicilios de los encuestados. La aplicación se desarrolló del 04 al 29 de mayo de 2020. Los datos fueron tabulados en una matriz Excel para su posterior análisis de los datos. Para ambas etapas de este estudio, se realizaron análisis de estadísticos descriptivos usando Software RStudio versión 4.0.

\section{Resultados}

El presente trabajo tuvo como primer objetivo caracterizar variables psicosociales y académicas durante el aislamiento físico por la Covid-19 de estudiantes de nivel primario y secundario del sur de Chile, específicamente aspectos como la conectividad, salud mental, actividades familiares y tareas escolares. El segundo objetivo fue analizar variables asociadas a la salud mental de padres y madres.

\subsection{Caracterización sociofamiliar en tiempos de aislamiento físico por Covid-19}

Respecto de las características sociofamiliares se recogió información de 7 variables. La primera pregunta realizada a las familias consultó por el parentesco de las personas que 
conviven con el estudiante, evidenciándose que la mayoría vivía sólo con la madre ( $\mathrm{N}=$ 3.013) y con ambos (padres y madres; $\mathrm{N}=2.989$ ) (cuadro 1).

Cuadro 1. Personas con las que vive el estudiante

\begin{tabular}{lrr}
\hline \multicolumn{1}{c}{$\mathbf{N}$} & $\mathbf{\%}$ \\
\hline Madre & 3.013 & $37 \%$ \\
Ambos (padres y madres) & 2.989 & $37 \%$ \\
Padre & 1.085 & $13 \%$ \\
Abuelo/a & 510 & $6 \%$ \\
Otro & 505 & $6 \%$ \\
\hline Total & 8.102 & $100 \%$ \\
\hline Fuente: Elaboración propia. & &
\end{tabular}

Fuente: Elaboración propia.

La segunda pregunta abordó el factor económico familiar, se consultó si alguien del grupo familiar se encontraba sin trabajo remunerado, donde $3.273(40 \%)$ declararon que sí, mientras que $4.829(60 \%)$ informaron que no. La tercera pregunta buscó especificar las personas responsables económicamente del núcleo familiar, para ellos se preguntó por la persona responsable de cubrir los gastos mensuales del hogar, declarándose en la mayoría de las familias 4.926 (61\%) era la madre, $1.539(19 \%)$ era el padre, y 1.637 (20\%) declararon otro.

La cuarta pregunta referida al ingreso económico promedio mensual del grupo familiar mostró que el principal rango de ingresos fluctúa entre $\$ 440$ y $\$ 750$ dólares $(\mathrm{N}=4.391)$, seguido del rango entre $\$ 190$ a $\$ 440(\mathrm{~N}=1.925)$ y del rango de $\$ 0$ a $190(\mathrm{~N}=1.547)$ (ver figura 1); los últimos dos promedios mensuales se incluyen dentro de la categoría de "sueldo mínimo por persona" establecido por familia en el país, lo cual dificulta la satisfacción de las necesidades básicas familiares en el contexto chileno actual.

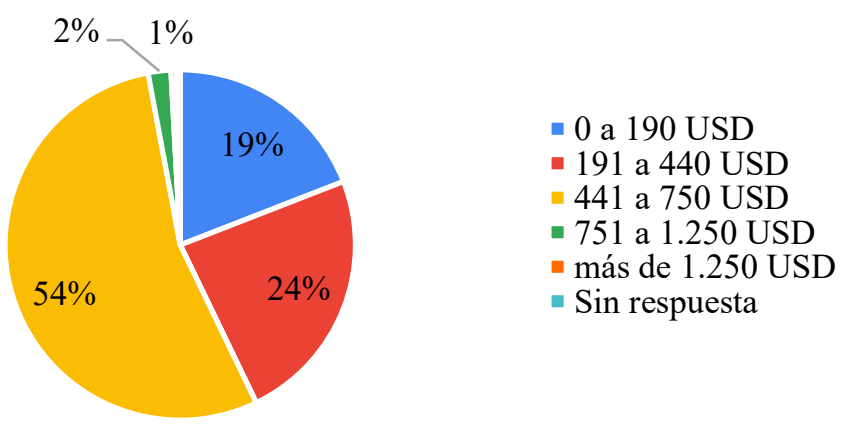

Figura 1. Ingreso económico mensual de grupo familiar en tiempo de aislamiento físico Fuente: Elaboración propia.

La quinta pregunta exploró si algún miembro del grupo familiar se encontraba enfermo, donde $3.216(40 \%)$ declararon tener algún miembro de la familia enfermo, mientras que 4.886 (60\%) declaró que no había ningún integrante enfermo. La sexta pregunta buscó especificar sobre el estado de salud familiar, por tanto, se consultó si alguno de los integrantes del grupo familiar tiene condición de enfermedad crónica, donde 3.111 (38\%) declaró que sí hay un enfermo crónico en la familia, mientras que 4.991 (62\%) respondió que no.

Finalmente, la séptima pregunta, consultó sobre las actividades que están desarrollando como familia en este periodo de aislamiento físico. La principal actividad desarrollada fue 
F. Sáez-Delgado, C. Olea-González, J. Mella-Norambuena, Y. López-Angulo, H. García-Vásquez, R. Cobo-Rendón y F. Sepúlveda López

ver televisión $(\mathrm{N}=5.478)$ y las actividades menos desarrolladas fueron karaoke $(\mathrm{N}=28)$ y cocinar $(\mathrm{N}=81)$ (figura 2$)$.

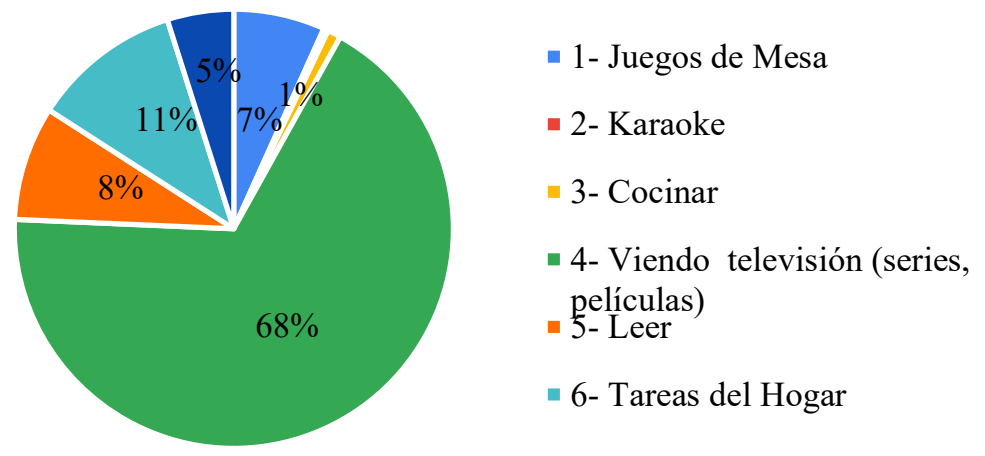

Figura 2. Actividades grupo familiar en tiempo de aislamiento físico Fuente: Elaboración propia.

\subsection{Conectividad durante aislamiento físico por Covid-19}

Para describir la conectividad, se hicieron tres preguntas a los participantes. Primeramente, si contaban con acceso a un computador en el hogar. De un total de 8.102 participantes, 4.788 respondieron afirmativamente, mientras que 3.314 respondieron negativamente. La segunda pregunta buscó profundizar en la primera, por tanto, se preguntó si el computador al que tienen acceso posee conexión a internet, siendo 3.637 participantes los que respondieron afirmativamente, mientras que 4.465 respondieron negativamente (figura 3).

\section{Acceso a Computador}

Acceso a Computador con Internet
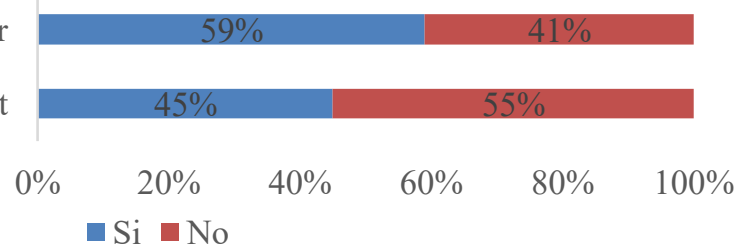

Figura 3. Acceso a computador e internet en el hogar

Fuente: Elaboración propia.

La tercera pregunta, corroboró el tipo de conexión a internet presente en el hogar, la principal categoría fue el plan de datos de celular $(\mathrm{N}=4.099)$, seguido de internet del hogar; mientras que, el menos usado es una red wifi-abierta $(\mathrm{N}=251)$ (figura 4 ). 


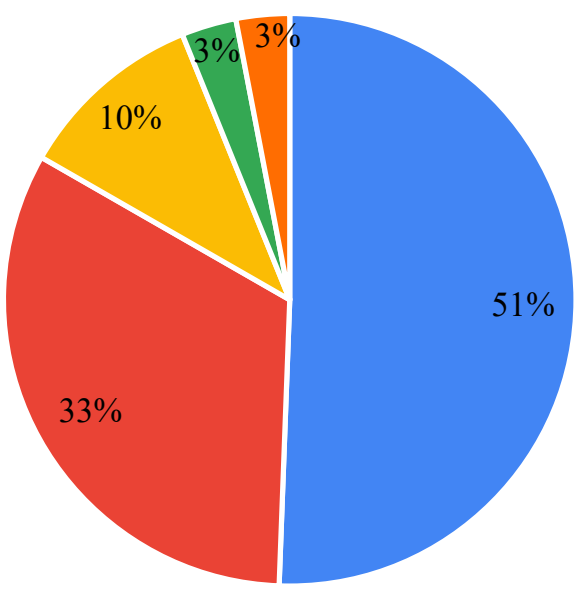

- 1.Plan de datos de celular

- 2.Plan internet hogar

$\because$ 3.Comparte internet con vecino/familiar

- 4. Se conecta a una red wifi abierta

$=5$. Otro

Figura 4. Tipo de conexión a internet en el hogar Fuente: Elaboración propia.

\subsection{Salud mental familiar durante aislamiento físico por Covid-19}

Para describir la salud mental de la familia se hicieron tres preguntas a los participantes. Primeramente, se les preguntó a los padres y madres si su hijo(a) se ha sentido con ansiedad, miedos nocturnos, mal humor o agresividad últimamente, donde 5.744 respondieron que sí, mientras que 1.362 respondieron que no y un número importante declaró tal vez $(\mathrm{N}=997)$.

En segundo lugar, se preguntó al padre, madre o responsable legal, si se ha sentido con ansiedad, miedos nocturnos, mal humor o agresividad en este último tiempo, donde 6.894 respondieron que sí, mientas que 380 respondieron que no. La tercera pregunta exploró si le gustaría que el psicólogo de la institución educativa los asesore cuando las condiciones lo permitan, donde 5.460 dijeron que sí, mientras que 2.398 dijeron que no (figura 5).

Respecto a la salud mental, ¿la/el estudiante se ha sentido con ansiedad, miedos nocturnos, mal humor o agresividad este último tiempo?

Usted como apoderado ¿se ha sentido con ansiedad, miedos nocturnos, mal humor o agresividad este último tiempo?

¿Le gustaría que el/a psicólogo/a del colegio lo asesore a usted como apoderado y al alumno cuando las condiciones lo permitan?

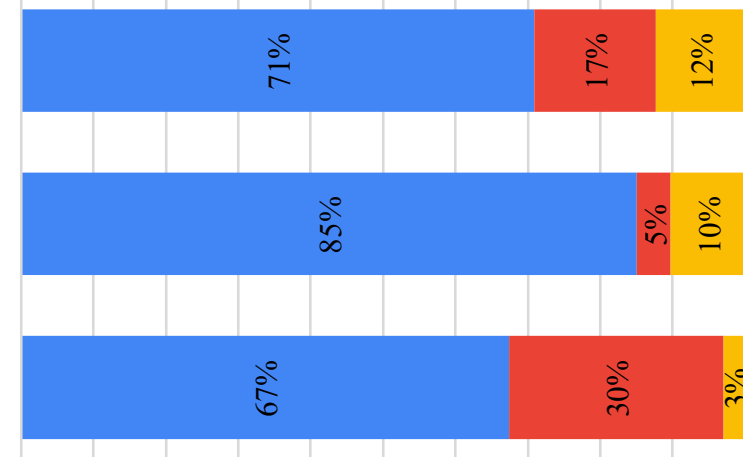

$\begin{array}{lllllllllll}0 \% & 10 \% & 20 \% & 30 \% & 40 \% & 50 \% & 60 \% & 70 \% & 80 \% & 90 \% & 100 \%\end{array}$

$\square \mathrm{Si} \quad \square$ No $\quad \square$ Tal Vez

Figura 5. Salud mental de padres, madres y estudiantes

Fuente: Elaboración propia. 


\subsection{Actividades académicas familiares en tiempos de aislamiento físico por Covid- 19}

Con respecto a la dimensión de tareas escolares, se exploraron aspectos como los hábitos de lectura, las dificultades que han presentado para la realización de las actividades escolares y el tiempo destinado para éstas por medio de cuatro preguntas. La primera consultó a los padres y madres si su hijo o hija ha podido realizar las tareas escolares, donde 5.104 respondieron que sí han podido realizarlas, mientras que 2.998 respondieron que no les ha sido posible.

La segunda pregunta buscó estimar en escolares, el tiempo diario destinado al estudio, donde estos declararon que 2.317 dedican 1 hora, 1.401 dedican 3 horas o más, y 4.384 que no tiene un tiempo destinado al estudio, es sólo cuando quieren (figura 6).
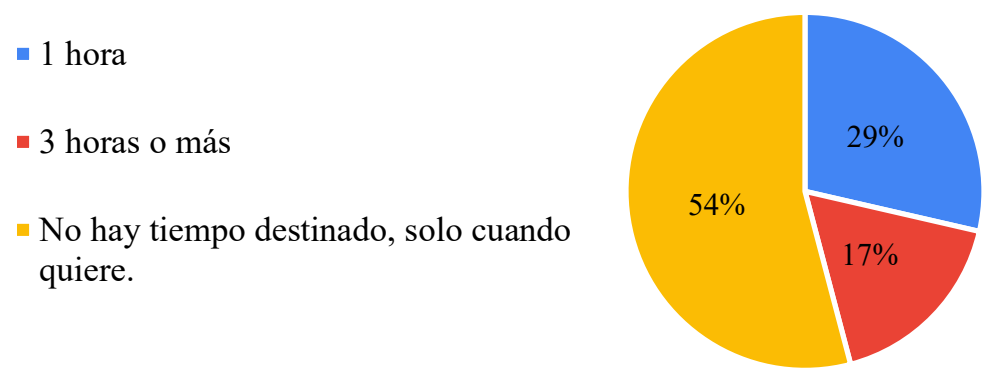

Figura 6. Tiempo dedicado al estudio durante el aislamiento físico Fuente: Elaboración propia.

La tercera pregunta buscó identificar la principal dificultad que habían tenido las familias para el trabajo con los materiales educativos entregados por los establecimientos educacionales, siendo 2.841 las familias que declaran poca motivación de las y los estudiantes, 2.829 manifestaron dificultades asociadas a la imposibilidad de imprimir el material y 1.401 explicitaron que el material pedagógico es poco claro, otras dificultades declaradas en menor medida fueron: no tener tiempo para supervisar el estudio de los y las hijas y no tener el espacio en el hogar adecuado para el estudio, las que se agruparon como "otro" (ver figura 7).
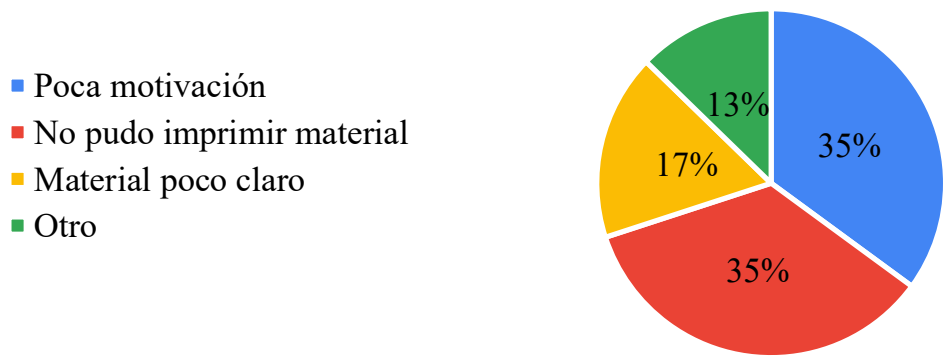

Figura 7. Dificultades de los estudiantes para la realización del material educativo Fuente: Elaboración propia.

La cuarta pregunta consultó sobre la frecuencia destinada como familia a la lectura, donde sólo 245 informó que la frecuencia de lectura era diaria, 972 informó una frecuencia de dos veces por semana, 2.511 informó que una vez al mes, y 4.374 informó que depende de otras 
actividades de la dinámica familiar como horas de trabajo de los padres y madres (ver figura 8$)$.

$$
\begin{aligned}
& \text { - Diaria } \\
& \text { - } 2 \text { Veces por semana } \\
& \text { - } 1 \text { vez al mes } \\
& \text { - Depende de otras actividades }
\end{aligned}
$$

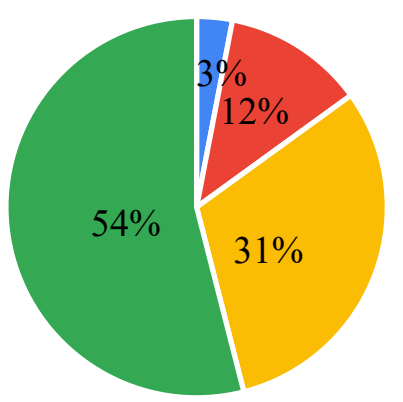

Figura 8. Frecuencia de lectura familiar durante el aislamiento físico Fuente: Elaboración propia.

\subsection{Descripción de variables de salud mental en padres y madres (ansiedad, depresión, estrés)}

Debido a la alta cantidad de respuestas relacionadas con dificultades de salud mental en los padres y madres, la segunda fase de este estudio buscó analizar variables asociadas a la salud mental de padres y madres, a través de la descripción de los niveles de ansiedad, depresión y estrés.

La Tabla 2 describe los promedios, desviación estándar, mínimo, máximo, rangos, simetría y curtosis de las tres escalas del DASS-2 1, considerando las escalas de respuesta de estos instrumentos de medición (0 a 3). La asimetría y curtosis para las escalas del DASS-2 1, se encuentran fuera del rango -1 a +1 , lo que indica variables con distribución estocástica.

Cuadro 2. Estadísticos descriptivos sobre las puntuaciones de las dimensiones del DASS21

\begin{tabular}{lccccccccc}
\hline & \multirow{2}{*}{ M } & \multirow{2}{*}{ DE } & \multirow{2}{*}{ MIN } & \multirow{2}{*}{ MAX } & \multirow{2}{*}{ RANGO } & \multicolumn{2}{c}{ ASIMETRÍA } & \multicolumn{2}{c}{ CURTOSIS } \\
& & & & & & $\mathbf{d t}$ & $\mathbf{B}$ & $\mathbf{d t}$ \\
\hline Depresión & 8,77 & 1,63 & 5,00 & 15,00 & 10,00 & 0,761 & 0,316 & 2,998 & 0,623 \\
Ansiedad & 10,38 & 2,21 & 5,00 & 20,00 & 15,00 & 1,434 & 0,316 & 5,891 & 0,623 \\
Estrés & 10,47 & 2,19 & 7,00 & 20,00 & 13,00 & 1,681 & 0,316 & 5,520 & 0,623 \\
\hline
\end{tabular}

Fuente: Elaboración propia.

$\mathrm{Al}$ analizar las puntuaciones de las medidas de salud mental de las y los participantes considerando los baremos presentados en la descripción del instrumento DASS-21, se identificó que en promedio la ansiedad se ubicó en nivel de extremadamente severa, y el estrés y la depresión fueron de nivel moderado.

\section{Discusión y conclusiones}

Este estudio tuvo por propósitos, (1) caracterizar variables psicosociales y académicas durante el aislamiento físico por la Covid-19 en familias de escolares de nivel primario y secundario del sur de Chile; y (2) analizar variables asociadas a la salud mental de padres y madres. 


\subsection{Características sociofamiliares en tiempos de aislamiento físico por Covid-19}

La caracterización de aspectos sociofamiliares en este estudio reveló que los escolares viven principalmente con su madre, que cerca de la mitad de las familias encuestadas tiene algún integrante que se encuentra sin trabajo remunerado e ingresos económicos mensuales insuficientes que pueden colocar a las familias en una situación vulnerable. Este resultado es relevante en relación con el aprendizaje de escolares. Un estudio en Nigeria reveló que el estado socioeconómico de los padres tuvo un impacto significativo en los aprendizajes de los escolares durante el cierre de las escuelas por la pandemia Covid-19 (Olaseni, 2020). Específicamente en Chile, existe evidencia de la desigualdad económica, siendo un tema latente en esta última década (Mieres, 2020); por lo que, el impacto de la pandemia está teniendo efectos negativos sobre la economía chilena lo que profundizaría los índices de desigualdad que existen en el país.

En cuanto a la salud familiar, algunas tienen miembros enfermos y enfermos crónicos, transformando la situación de aislamiento por la pandemia en una situación crítica. La literatura señala que en aquellos hogares donde existen condiciones de vulnerabilidad se pueden presentar distintos factores de riesgo (cambios en las rutinas y dinámicas familiares, pérdida de empleos de los padres y madres o enfermedades) los cuales generan un impacto desfavorable en las prácticas de crianza limitando las oportunidades de estimulación de escolares y de respuestas adecuadas a sus demandas (Prats et al., 2018). $\mathrm{El}$ apoyo que brindan las familias en temas académicos a escolares, se asocian directamente con el desempeño y los logros académicos de éstos (Martínez, Torres y Ríos, 2020). Por lo tanto, urge desarrollar acciones de apoyo, que impacten positivamente en la salud de las familias, con el objetivo de prevenir un efecto paralelo en variables académicas y de salud mental de los y las escolares. Las familias declararon que la principal actividad que están desarrollando en este período de aislamiento físico fue ver series y películas en televisión, sin embargo, la actividad académica de leer, sólo se realizó mínimamente. Esto es consistente con estudios previos que han mostrado una disminución drástica en el plan de lectura de escolares durante el aislamiento físico (Olaseni, 2020) y consecuentemente tal como es sugerido por Ceballos (2020) es posible hipotetizar prospectivamente una disminución de las habilidades de comprensión lectora.

En definitiva, es recomendable que los establecimientos educativos promuevan acciones que integren a las familias para que se logre una concientización y compromiso en la formación académica de sus hijos e hijas ( Martínez, Torres y Ríos, 2020). Con la situación extraordinaria de aislamiento físico de hoy por la Covid-19, se vuelve indispensable proporcionar a las familias herramientas para lidiar con la crisis. Específicamente se requiere la entrega de orientaciones que promuevan la creación de ambientes familiares positivos para amortiguar los problemas de salud mental, disfunción familiar y desajuste emocional de los y las estudiantes.

\subsection{Conectividad durante aislamiento físico por Covid-19}

La conectividad en tiempos de pandemia se ha vuelto un requerimiento crucial para recibir las guías de aprendizaje, la comunicación con compañeros y docentes en el desarrollo de actividades de estudio y mantener la interacción social facilitadora de aprendizajes. Sin embargo, los resultados de la presente investigación mostraron que prácticamente la mitad de las familias no tiene computador ni acceso a internet. Esto es consistente con los resultados de una investigación en el contexto mexicano que identificó que las principales problemáticas que afrontan escolares son de tipo tecnológicos (Sánchez et al., 2020). Los 
obstáculos más comunes que surgen durante la implementación del aprendizaje en línea son el acceso limitado a internet y la poca experiencia de los aprendizajes en ambientes virtuales tanto en profesores como en estudiantes (Gunawan y Fathoroni, 2020; RamosHuenteo et al., 2020).

Aunque se propuso el aprendizaje en línea como una respuesta positiva al período de cierre de escuelas por Covid-19, esta medida tiene grandes limitaciones puesto que no se puede asegurar que todos los y las estudiantes tengan acceso a computadoras y a conexión de internet (Mulenga y Marbán, 2020). Indiscutiblemente, los estudiantes que viven en situación de desventaja económica estarán sujetos a una variedad de barreras que incluyen desconexión educativa a largo plazo, exclusión digital, baja gestión de la tecnología y mayores desafíos psicosociales (Mailizar, Maulina y Bruce, 2020). La literatura ha señalado que escolares que no pueden acceder a los recursos digitales facilitados por las escuelas podrían quedarse atrás académicamente en relación con aquellos que sí tengan acceso (Frenette, Frank y Deng, 2020).

El acceso a equipos informáticos, conexiones de alta velocidad, entre otros recursos tecnológicos se han convertido en los mediadores de la experiencia educativa. Como consecuencia se han enfatizado las diferencias sociales previamente existentes para grupos vulnerables. La apertura de una brecha virtual lleva a la discusión sobre los desafíos de mantener condiciones democráticas e igualitarias en las escuelas (Almazán, 2020).

En relación con esta problemática, las políticas educativas tanto previo a la pandemia Covid-19 como durante la pandemia han sido insuficientes y aún son muy superficiales para promover el mejor desarrollo de las actividades académicas. Se requiere con urgencia acciones que mitiguen estas diferencias y faciliten los aprendizajes de las y los estudiantes (Sintema, 2020; Zhou et al., 2020). Es impostergable transitar de acciones remediales e inmediatas durante la pandemia como alternativa a corto plazo a aquellas que sean sostenibles en el tiempo para disminuir las brechas tecnológicas mencionadas e impactar en la disminución de las injusticias sociales existentes.

\subsection{Actividades académicas durante el aislamiento físico por Covid-19}

Este estudio da cuenta que algunos escolares no han podido realizar sus tareas académicas, que la mayoría no tiene un tiempo fijo destinado al estudio, y que la principal dificultad para la realización del material educativo fue la baja motivación y no tener posibilidad de imprimir el material. Respecto de la lectura familiar la mayoría no tiene un horario establecido y depende de otras actividades propias de las dinámicas familiares.

Estos hallazgos son consistentes con estudios previos que demostraron que la pandemia realmente ha tenido un impacto negativo en los procesos de estudio y aprendizajes de escolares, ya que muchos no están acostumbrados a aprender de manera efectiva por sí mismos ( Owusu, Koomson y Hanson, 2020). La implementación del aprendizaje en línea durante la pandemia Covid-19 es una barrera que ha mostrado un impacto negativo en los aprendizajes (Mailizar, Maulina y Bruce, 2020); siendo de especial importancia que las familias promuevan conductas de estudio a partir de las orientaciones y sugerencias de las instituciones educativas.

La pérdida de horas de contacto e interacción generada en las dinámicas de presencialidad entre profesores y estudiantes debe ser ahora compensada por la reestructuración y esfuerzo de corregulación de las dinámicas familiares para generar instancias de apoyo que permitan facilitar el logro de objetivos de aprendizaje de las y los escolares. Es 
recomendable para las familias implementar estrategias potenciadoras del desarrollo psicoeducativo, siendo clave la regulación del uso del tiempo entre actividades de ocio y académicas; además de fomentar la motivación por el aprendizaje.

\subsection{Caracterización de la salud mental de familias de escolares durante aislamiento físico por Covid-19}

El estado de alerta generado por la Covid-19 y todas sus consecuencias suponen un conjunto de estresores nuevos y diferentes a otros anteriores que puede provocar alteraciones psicológicas en la población de escolares y sus familias.

La primera etapa de este estudio constató que la mayoría de las y los escolares y sus padres y madres han mostrado dificultades en el estado de ánimo, miedos nocturnos, mal humor o agresividad. La segunda etapa del estudio confirmó niveles extremos de ansiedad y moderados de estrés y depresión de los padres y madres encuestados. Al comparar estos resultados con los obtenidos en otras muestras de adultos -expuestos a medidas de confinamiento por el coronavirus- se observó diferencias, siendo menores las puntuaciones reportadas en países como China, Singapur e India (Chew et al., 2020; Wang et al., 2020).

Estos resultados se podrían explicar por dos aspectos señalados en la literatura: la temporalidad de medición respecto del inicio de la pandemia y la cantidad de mujeres participantes en la muestra recolectada. Respecto de la temporalidad, un estudio español describe que la presencia de puntuaciones bajas son posibles de encontrarlas al principio de la alarma, en tal sentido, se prevé que la sintomatología aumentará según vaya transcurriendo el confinamiento (Ozamiz-Etxebarria et al., 2020), lo que se podría asociar a este resultado puesto que el presente estudio obtuvo las medidas de los padres y madres finalizando el mes de mayo del 2020, es decir, luego de 60 días del primer caso reportado en Chile. La presencia de sentimientos de inseguridad e incertidumbre relacionadas con la pandemia y sumado a la falta de claridad sobre el desarrollo de esta enfermedad, facilita la aparición de conductas de ansiedad (Gallegos et al., 2020). Respecto de la muestra, es importante destacar que diversos estudios refieren que la presencia de mayores puntuaciones en los niveles de ansiedad, depresión y estrés han sido reportados en participantes mujeres (Mazza et al., 2020; Wang et al., 2020), en el caso del presente estudio, el 93\% de la información analizada corresponde a mujeres, lo que podría explicar los hallazgos encontrados.

A pesar de las iniciativas emergentes y provisorias desplegadas para minimizar las consecuencias de las medidas tomadas por la Covid-19 en salud mental, estas no han mostrado la efectividad esperada considerando los resultados de este estudio, donde se vuelve a confirmar el impacto negativo de los efectos de la pandemia en la salud mental de integrantes de las comunidades educativas en Chile (Ramos-Huenteo et al., 2020). Esta situación se evidencia en población con altos índice de vulnerabilidad (JUNAEB, 2020), por tanto, se reiteran nuevas injusticias sociales en la población más desaventajada. Prestar atención sostenida a la salud mental de las personas ante la emergencia por Covid19 implica articular estrategias efectivas a nivel macro y micro social que garanticen el óptimo estado de las familias sin distinción, desafío que aspira el logro del bienestar de las personas.

En definitiva, la actual situación de pandemia ha afectado a todas las personas, pero, sin duda la población con más altos índices de vulnerabilidad, caracterizada por la inestabilidad laboral, bajos ingresos económicos, escasos recursos tecnológicos y enfermedades, han sufrido más fuerte las consecuencias. Se debe considerar que, a sus 
preocupaciones actuales, se han sumado todas las asociadas a los efectos de la pandemia por coronavirus. Se podría pronosticar que la actual situación de pandemia es un factor que contribuye a profundizar los problemas de salud mental en las familias, implicando mayores brechas sociales.

\section{Limitaciones}

En la primera parte de este estudio, se utilizó una encuesta ad-hoc sobre componentes sociofamiliares. Al respecto, se requieren investigaciones que implementen mediciones con escalas sobre variables específicas de los componentes de esta investigación para avanzar en el alcance del estudio y comprensión del impacto de la Covid-19 en las comunidades educativas. Si bien se obtuvo información en escolares, el instrumento fue respondido por las familias, por tanto, se necesita un estudio directamente aplicando instrumentos de medida en los y las estudiantes. En la segunda parte del estudio, la principal limitación estuvo en el tamaño de la muestra. Si bien los resultados son un aporte relevante, es importante un estudio que incluya una muestra mayor para tener más representatividad de la población.

\section{Orientaciones para la futura investigación}

Sería interesante realizar estudios en profesores y estudiantes directamente para conocer cuál es el impacto del aislamiento físico por Covid-19, lo que permitirá robustecer los antecedes para ponerlos a disposición de las instituciones académicas e investigadores para el desarrollo de intervenciones de acuerdo con las necesidades y problemáticas identificadas, considerando que gran parte de la problemática de la enfermedad se puede impedir cambiando comportamientos de las familias y donde la psicología puede aportar a explicar, prevenir e intervenir para su respectiva solución (Urzúa et al., 2020). Se sugiere la realización de nuevos estudios que profundicen en las estrategias desarrolladas por promover el aprendizaje y atender la educación socioemocional (Cervantes y Gutiérrez, 2020).

\section{Agradecimientos}

Esta investigación fue financiada por el Fondo FAA 01/2020 de la Dirección de Investigación de la Universidad Católica de la Santísima Concepción.

\section{Referencias}

Almazán, A. (2020). Covid-19: ¿Punto sin retorno de la digitalización de la educación? Revista Internacional de Educación para la Justicia Social, 9(3), 1-4.

Ang, K. y Loh, P. (2019). Mental health and coping in parents of children with autism spectrum disorder (ASD) in Singapore: An examination of gender role in caring. Journal of Autism and Developmental Disorders, 49, 2129-2 145. https://doi.org/10.1007/s 10803-019-03900-w

Antúnez, Z. y Vinet, E. (2012). Escalas de depresión, ansiedad y Estrés (DASS-21): Validación de la versión abreviada en estudiantes universitarios chilenos. Terapia Psicológica, 30(3), 49-55. http://doi.org/10.4067/S07 18-48082012000300005

Ato, M., López, J. y Benavente, A. (2013). Un sistema de clasificación de los diseños de investigación en psicología. Anales de Psicología, 29(3), 1038-1059. https://doi.org/10.6018/analesps.29.3.178511 
Bao, W. (2020). Covid-19 and online teaching in higher education: A case study of Peking University. Human Behavior and Emerging Technologies, 2, 113-115. https://doi.org/10.1002/hbe2.191

Cáceres, K. (2020). Educación virtual: Creando espacios afectivos de convivencia y aprendizaje en tiempos de Covid-19. CienciAmérica, 9(2), 1-7. https://doi.org/10.33210/ca.v9i2.284

Ceballos, N. (2020). El efecto del aislamiento social por el Covid-19 en la conciencia emocional y en la comprensión lectora. Estudio sobre la incidencia en alumnos con trastornos de aprendizaje y menor acceso a las nuevas tecnologías. Revista Internacional de Educación para la Justicia Social, 9(3), 1-13.

Chew, N., Lee, G., Tan, B., Jing, M., Goh, Y., Ngiam, N. y Sharma, A. (2020). A multinational, multicentre study on the psychological outcomes and associated physical symptoms amongst healthcare workers during Covid-19 outbreak. Brain, 88, 559-565. https://doi.org/10.1016/j.bbi.2020.04.049

Cervantes, E. y Gutiérrez, P. (2020). Resistir la Covid-19. Intersecciones en la educación de Ciudad Juárez, México. Revista Internacional de Educación para la Justicia Social, 9(3), 7-23. https://doi.org/10.15366/riejs2020.9.3.001

Frenette, M., Frank, K. y Deng, Z. (2020). School closures and the online preparedness of children during the Covid-19 pandemic economic insights school closures and the online preparedness of children during the Covid-19 pandemic. Economic Insights, 1(103), 1-11.

Gallegos, M., Zalaquett, C., Luna Sanchez, S. E., Mazo-Zea, R., Ortiz-Torres, B., Penagos-Corzo, J., ... y Polanco, F. (2020). Cómo afrontar la pandemia del coronavirus (Covid-19) en las Américas: Recomendaciones y líneas de acción sobre salud mental. Revista Interamericana de Psicología, 54(1), 1-28. https://doi.org/10.30849/ripijp.v54i1.1304

Gilbert, L., Spears, C. y Mistry, R. (2017). Latino immigrant parents' financial stress, depression, and academic involvement predicting child academic success. Psychology in the Schools, 54(9), 1202-1215. https://doi.org/10.1002/pits.22067

Gunawan, M. Y. y Fathoroni. (2020). Variations of models and learning platforms for prospective teachers during the Covid-19 pandemic period. Indonesian Journal of Teacher Education, 1(2), 61-70.

Holguín, E. y Sandoval, P. (2020). Resistir la Covid-19. Intersecciones en la educación de Ciudad Juárez, México. Revista Internacional de Educación para la Justicia Social, 9(3), 7-23. https://doi.org/10.15366/riejs2020.9.3.001

JUNAEB. (2020). Índice de vulnerabilidad escolar 2020. JUNAEB.

Lui, M., Lau, G., Tam, V., Chiu, H., Li, S. y Sin, K. (2019). Parents' impact on children's school performance: Marital satisfaction, parental involvement, and mental health. Journal of Child and Family Studies, 29,1548-1560. https://doi.org/10.1007/s 10826-019-01655-7

Lorenzo-Blanco, E., Meca, A., Unger, J., Romero, A., Gonzales-Backen, M., Piña-Watson, B., ... y Villamar, J. (2016). Latino parent acculturation stress: Longitudinal effects on family functioning and youth emotional and behavioral health. Journal of Family Psychology, 30(8), 966-976. https://doi.org/10.1037/famo000223

Lusquiños, C. (2020). Acceso a TIC, habitualidad en el uso y desempeño escolar en contextos diferenciados ¿Una alternativa para el aprendizaje en escuelas primarias?. Revista Internacional de Educación para la Justicia Social, 9(3), 1-15.

Mailizar, A., Maulina, S. y Bruce, S. (2020). Secondary school mathematics teachers' views on elearning implementation barriers during the Covid-19 pandemic: The case of Indonesia. 
Journal of Mathematics, Science and Technology Education, 16(7), 1-9.

https://doi.org/10.29333/ejmste/8240

Martínez, G., Torres, M. y Ríos, V. (2020). El contexto familiar y su vinculación con el rendimiento académico. IE Revista de Investigación Educativa, 1 1(e627), 1-17.

https://doi.org/10.33010/ie_rie_rediech.v11io.657

Mazza, C., Ricci, E., Biondi, S., Colasanti, M., Ferracuti, S., Napoli, C. y Roma, P. (2020). A nationwide survey of psychological distress among italian people during the Covid-19 pandemic: Immediate psychological responses and associated factors. International Journal of Environmental Research and Public Health, 17(9), 1-14. https://doi.org/10.3390/ijerph 17093165

Mieres, M. (2020). Develando los determinantes de la desigualdad del ingreso en Chile: Estudio empírico regional. Revista de Análisis Económico, 35(1), 99-127. https://doi.org/10.4067/s07 18-88702020000100099

MINSAL. (2020). Ministerio de Salud confirma primer caso de coronavirus en Chile. https://www.minsal.cl/ministerio-de-salud-confirma-primer-caso-de-coronavirus-enchile/

Murillo, F. J. (2016). Midiendo la segregación escolar en América Latina. Un análisis metodológico utilizando el TERCE. REICE. Revista Iberoamericana sobre Calidad, Eficacia y Cambio en Educación, 14(4), 33-60. https://doi.org/10.15366/reice2016.14.4.002

Mulenga, E. y Marbán, J. (2020). Is Covid-19 the gateway for digital learning in mathematicseducation. Contemporary Educational Technology, 12(2), 1-11. https://doi.org/10.30935/cedtech/7949

Olaseni, A. (2020). Covid-19 pandemic: Impact of socio-demographic factors and parent's life orientation on enforced learning in pupils during lock-down in Nigeria. Cape Comorin, 2(4), 34-39.

Orellana, C. y Orellana, L. (2020). Predictors of emotional symptoms during residential lockdown due to the Covid-19 pandemic in El Salvador. Actualidades en Psicología, 34(128), 103-120. https://doi.org/10.15517/ap.v34i128.41431

Owusu, C., Koomson, C. y Hanson, D. (2020). The impact of Covid-19 on learning-the perspective of the Ghanaian student. European Journal of Education Studies, 7(3), 88-101.

https://doi.org/10.5281/zenodo.3753586

Ozamiz-Etxebarria, N., Dosil-Santamaria, M., Picaza-Gorrochategui, M. y Idoiaga-Mondragon, N. (2020). Niveles de estrés, ansiedad y depresión en la primera fase del brote del Covid-19 en una muestra recogida en el norte de España. Cadernos de Saúde Pública, 36(4), 1-10. https://doi.org/10.1590/0102-311X00054020

Pierre, R. y Harris, P. (2020). Covid-19 in Latin America: Challenges and opportunities. Revista Chilena de Pediatria, 91(2), 179-182. https://doi.org/10.32641/rchped.vi91i2.2157

Prats, L., Segretin, M., Fracchia, C., Giovannetti, F., Mancini, N. y Lipina, S. (2018). Desarrollo cognitivo infantil y prácticas maternas de crianza: Implementación de una intervención con madres y niños de hogares con necesidades básicas insatisfechas (NBI). Revista Latinoamericana de Ciencias Psicológica, 10(1), 1-45. https://doi.org/10.5872/psiencia/10.1.24

Quiroz, C. (2020). Pandemia Covid-19 e inequidad territorial: El agravamiento de las desigualdades educativas en Chile. Revista Internacional de Educación para la Justicia Social, 9(3), 1-6.

Rajkumar, R. (2020). Covid-19 and mental health: A review of the existing literature. Asian Journal of Psychiatry, 52, 1-5. https://doi.org/10.1016/j.ajp.2020.102066 
F. Sáez-Delgado, C. Olea-González, J. Mella-Norambuena, Y. López-Angulo, H. García-Vásquez,

R. Cobo-Rendón y F. Sepúlveda López

Ramos-Huenteo, V., García-Vásquez, H., Olea-González, C., Lobos-Peña, K. y Sáez-Delgado, F. (2020). Percepción docente respecto al trabajo pedagógico durante la Covid-19. CienciAmérica, 9(2), 334-353. https://doi.org/10.33210/ca.v9i2.325

Rodicio-García, M., Ríos-de-Deus, M., Mosquera-González, M. y Abilleira, M. (2020). La brecha digital en estudiantes españoles ante la crisis de la Covid-19. Revista Internacional de Educación para la Justicia Social, 9(3), 103-125. https://doi.org/10.15366/riejs2020.9.3.006

Sánchez, M., Martínez, A., Torres, R., de Agüero, M., Hernández, A., Benavides, M., Rendón, V. y Jaimes, C. (2020). Retos educativos durante la pandemia de Covid-19: Una encuesta a profesores de la UNAM. Revista Digital Universitaria, 21(3), 1-24. http://doi.org/10.22201/codeic.16076079e.2020.v21n3.a12

Sintema, E. (2020). Effect of Covid-19 on the performance of grade 12 students: Implications for STEM education. Eurasia Journal of Mathematics, Science and Technology Education, 16(7), 16. https://doi.org/10.29333/ejmste/7893

Tawfik, A., Reeves, T. y Stich, A. (2016). Intended and unintended consequences of educational technology on social inequality. TechTrends, 60(6), 598-605. https://doi.org/10.1007/s1 1528-016-0109-5

Tedesco, J. (2014). Tecnologías de la información y desigualdad educativa en América Latina. Archivos Analíticos de Políticas Educativas, 22(48), 1-15. http://dx.doi.org/10.14507/epaa.v22n48.2014.

Urzúa, A., Vera-Villarroel, P., Caqueo-Urízar, A. y Polanco-Carrasco, R. (2020). La psicología en la prevención y manejo del Covid-19. Aportes desde la evidencia inicial. Terapia Psicológica, 38(1), 103-118. http://doi.org/10.4067/So718-48082020000100103_

Van Lancker, W. y Parolin, Z. (2020). Covid-19, school closures, and child poverty: A social crisis in the making. The Lancet Public Health, 5(5), e243-e244.

https://doi.org/10.1016/S2468-2667(20)30084-O

Villafuerte, J., Bello, J., Pantaleón, Y. y Bermello, J. (2020). Rol de los docentes ante la crisis del covid-19, una mirada desde el enfoque humano. Revista Electrónica Formación y Calidad Educativa, 8(1), 134-150.

Viner, R., Russell, S., Croker, H., Packer, J., Ward, J., Stansfield, C., Mytton, O., Bonell, C. y Booy, R. (2020). School closure and management practices during coronavirus outbreaks including Covid-19: a rapid systematic review. The Lancet Child and Adolescent Health, 4(5), 397-404. https://doi.org/10.1016/S2352-4642(20)30095-X

Wang, C., Pan, R., Wan, X., Tan, Y., Xu, L., McIntyre, R., Chooe, F., Tranc, B., Hoe, R., Sharmah, V. y Ho, C. (2020). A longitudinal study on the mental health of general population during the Covid-19 epidemic in China. Brain, Behavior, and Immunity, 87, 40-48. https://doi.org/10.1016/j.bbi.2020.04.028

Wu, M., Xu, W., Yao, Y., Zhang, L., Guo, L., Fan, J. y Chen, J. (2020). Mental health status of students' parents during COVID-19 pandemic and its influence factors. General Psychiatry, 33(4), 1-9. https://doi.org/10.1136/gpsych-2020-100250

Xie, Z. y Yang, J. (2020). Autonomous learning of elementary students at home during the Covid19 epidemic: A case study of the second elementary school in Daxie, Ningbo, Zhejiang Province, China. Best Evidence of Chinese Education, 4(2), 535-541. https://doi.org/10.15354/bece.20.rpoo9

Yi, Y., Lagniton, P., Ye, S., Li, E. y Xu, R. (2020). Covid-19: What has been learned and to be learned about the novel coronavirus disease. International Journal of Biological Sciences, 16(10), 1753-1766. https://doi.org/10.7150/ijbs.45134 
Zolotov, Y., Reznik, A., Bender, S. y Isralowitz, R. (2020). Covid-19 fear, mental health, and substance use among Israeli university students. International Journal of Mental Health and Addiction, 10, 1-7. https://doi.org/10.1007/s1 1469-020-00351-8

Zhou, L., Wu, S., Zhou, M. y Li, F. (2020). "School's out, but class' on", The largest online education in the world today: Taking China's practical exploration during the Covid-19 epidemic prevention and control as an example. SSRN Electronic Journal, 4(2), 501-519.

\section{Breve CV de los autores}

\section{Fabiola Sáez-Delgado}

Académica investigadora del Departamento Fundamentos de la Pedagogía de la Facultad de Educación de la Universidad Católica de la Santísima Concepción, Chile. Estudios profesionales de Profesora, Licencia en Educación, Magíster en Educación y Doctora en Psicología por la Universidad de Concepción, Chile. Su línea de investigación son las variables cognitivas motivacionales de los procesos de enseñanza y aprendizaje; también las variables de salud mental en comunidades educativas. Cátedras en el Programa de Doctorado en Educación (UCSC) sobre Investigación en Educación y Análisis cuantitativo de datos con uso de Software estadístico R. Se ha especializado en revisiones sistemáticas de la literatura, psicometría y análisis de modelos predictivos y explicativos en Educación. Miembro del Grupo de Investigación Internacional Sociedad, Educación y Psicología (GISEP). ORCID ID: https://orcid.org/o000-0002-7993-5356. Email: fsaez@ucsc.cl

\section{Constanza Olea-González}

Licenciada en Historia y Educación, Profesora de Historia y Geografía en Educación Media por la Universidad Católica de la Santísima Concepción. Diplomada en Evaluación de los Aprendizajes por la Universidad Andrés Bello. Actualmente es estudiante del Programa de Magíster en Ciencias de la Educación en la Universidad Católica de la Santísima Concepción y laboralmente desempeña funciones como Coordinadora de la Unidad Técnico-Pedagógica del Liceo Polivalente Las Salinas dependiente de la Dirección de Administración Municipal de Educación de Talcahuano. Su línea de investigación es la autorregulación del aprendizaje, la persistencia académica y la intención de abandono en estudiantes de educación secundaria. ORCID ID: https://orcid.org/O000-0001-72084870. Email: colea@magisteredu.ucsc.cl

\section{Javier Mella-Norambuena}

Docente del Departamento de Ciencias la Universidad Técnica Federico Santa María, Concepción, Chile. Es profesor, Licenciado en Educación y Magíster en Psicología de la Salud por la Universidad de Concepción, Chile. Su línea de investigación son las variables cognitivomotivacionales de los procesos de enseñanza y aprendizaje; y las variables de salud mental en comunidades educativas. Se ha especializado en revisiones sistemáticas de la literatura, metodología de la investigación, y análisis de datos cuantitativos con software SPSS, RStudio y Python. Miembro del Grupo de Investigación Internacional Sociedad, Educación y Psicología (GISEP). ORCID ID: https://orcid.org/OOOO-0002-4288-142X. Email: javier.mellan@usm.cl

\section{Yaranay López-Angulo}

Licenciada en Psicología 2013, Máster Psicología Educativa 2018, La Habana, Cuba. Dra. (c) del Programa de Doctorado en Psicología de la Universidad de Concepción, Chile. Académica y profesora asistente de la Universidad Santo Tomás, Concepción, Chile. Líneas de investigación: variables cognitivas-motivacionales relacionadas al abandono universitario, psicoeducación y género. Profesora de: Psicología Educativa, Evolutiva, Metodología y Análisis de Datos. Últimas publicaciones: Apoyo social, sexo y área del conocimiento en el rendimiento académico 
autopercibido de estudiantes universitarios chilenos. Formación universitaria, 13(3), 11-18. Revisión sistemática sobre instrumentos de autorregulación del aprendizaje en estudiantes de educación secundaria. Información Tecnológica, 31(4), 85-98. ORCID ID: https://orcid.org/OOoOooo2-3331-6875. Email: ylopez7@santotomas.cl

\section{Héctor García-Vásquez}

Estudios profesionales de Ingeniero Mecánico en Producción Industrial por la Universidad Tecnológica de Chile (INACAP). Realizó un postítulo sobre Pedagogía para docentes profesionales y técnicos de Educación Superior de la Universidad de Concepción, Chile. Actualmente es estudiante en el Programa de Magíster en Ciencias de la Educación de la Universidad Católica de la Santísima Concepción, Chile. Laboralmente es docente del área industrial en el Liceo Industrial de Concepción, Chile. Su línea de Investigación son los mecanismos de autorregulación en estudiantes de la educación técnico profesional. ORCID ID: https://orcid.org/OOOO-0001-96544034. Email: hgarcia@magisteredu.ucsc.cl

\section{Rubia Cobo-Rendón}

Es Psicóloga y Magister Scientiarum en Psicología Clínica por la Universidad Rafael Urdaneta, Venezuela. Doctora en Psicología por la Universidad de Concepción, Chile. Docente en la Facultad de Psicología en la Universidad de San Sebastián, sede Concepción. Investigadora en el Laboratorio de Investigación e Innovación Educativa de la Dirección de Docencia de la Universidad de Concepción. Líneas de investigación: bienestar, salud mental, fortalezas del carácter, procesos enseñanza aprendizaje en Educación Superior, abandono y adaptación a la vida universitaria. Miembro de la Sociedad Científica de Psicología de Chile (SCP) y la Sociedad Venezolana de Psicología Positiva (SOVEPPOS) ORCID ID: https://orcid.org/O000-0002-3350-071X. Email: rcobor@docente.uss.cl; rubiacobo@udec.cl

\section{Felipe Sepúlveda López}

Académico Asociado de la Facultad de Educación de la Universidad Católica de la Santísima Concepción, jefe del Departamento de Fundamentos de la Pedagogía y director del Centro de Investigación en Educación y Desarrollo. Estudios profesionales de Biología Marina, Magister en Ciencias del Mar (Universidad Católica del Norte), Magister en Educación (Universidad de Texas, EE. UU.), Doctor en Liderazgo y Administración Educativa (Mary-Hardin Baylor, EE. UU.). Durante 2006 al 2014 se desempeñó como profesor de primaria del sistema público escolar en la ciudad de Dallas, Texas-EE. UU. enseñando en un programa bilingüe a estudiantes de familias inmigrantes que viven en contextos de vulnerabilidad social. Colabora en iniciativas de investigación asociadas al liderazgo escolar y aprendizaje de lectura/matemáticas a nivel escolar primario. ORCID ID: https://orcid.org/OOOO-0001-5703-383X. Email: fsepulveda@ucsc.cl 